\title{
Spatial Attention, Motor Intention, and Bayesian Cue Predictability in the Human Brain
}

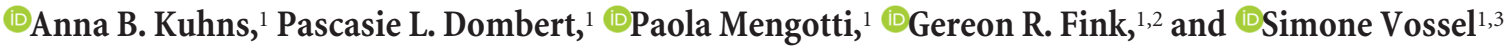 \\ ${ }^{1}$ Cognitive Neuroscience, Institute of Neuroscience and Medicine (INM-3), Research Centre Juelich, 52425 Juelich, Germany, ${ }^{2}$ Department of Neurology, \\ University Hospital Cologne, 50937 Cologne, Germany, and ${ }^{3}$ Department of Psychology, University of Cologne, 50931 Cologne, Germany
}

Predictions about upcoming events influence how we perceive and respond to our environment. There is increasing evidence that predictions may be generated based upon previous observations following Bayesian principles, but little is known about the underlying cortical mechanisms and their specificity for different cognitive subsystems. The present study aimed at identifying common and distinct neural signatures of predictive processing in the spatial attentional and motor intentional system. Twenty-three female and male healthy human volunteers performed two probabilistic cueing tasks with either spatial or motor cues while lying in the fMRI scanner. In these tasks, the percentage of cue validity changed unpredictably over time. Trialwise estimates of cue predictability were derived from a Bayesian observer model of behavioral responses. These estimates were included as parametric regressors for analyzing the BOLD time series. Parametric effects of cue predictability in valid and invalid trials were considered to reflect belief updating by precision-weighted prediction errors. The brain areas exhibiting predictability-dependent effects dissociated between the spatial attention and motor intention task, with the right temporoparietal cortex being involved during spatial attention and the left angular gyrus and anterior cingulate cortex during motor intention. Connectivity analyses revealed that all three areas showed predictability-dependent coupling with the right hippocampus. These results suggest that precision-weighted prediction errors of stimulus locations and motor responses are encoded in distinct brain regions, but that crosstalk with the hippocampus may be necessary to integrate new trialwise outcomes in both cognitive systems.

Key words: attentional networks; Bayesian inference; fMRI; motor preparation; psychophysiological interaction

\section{Significance Statement}

The brain is able to infer the environments' statistical structure and responds strongly to expectancy violations. In the spatial attentional domain, it has been shown that parts of the attentional networks are sensitive to the predictability of stimuli. It remains unknown, however, whether these effects are ubiquitous or if they are specific for different cognitive systems. The present study compared the influence of model-derived cue predictability on brain activity in the spatial attentional and motor intentional system. We identified areas with distinct predictability-dependent activation for spatial attention and motor intention, but also common connectivity changes of these regions with the hippocampus. These findings provide novel insights into the generality and specificity of predictive processing signatures in the human brain.

\section{Introduction}

How we perceive and respond to our environment does not depend solely on the sensory input that we receive, but also on predic-

Received 0ct. 20, 2016; revised April 10, 2017; accepted April 17, 2017.

Author contributions: A.B.K., P.L.D., P.M., G.R.F., and S.V. designed research; A.B.K. performed research; A.B.K. and S.V. analyzed data; A.B.K., P.L.D., P.M., G.R.F., and S.V. wrote the paper.

This work was supported by the Federal Ministry of Education and Research (BMBF Grant $01 G 01401$ to S.V.). We thank our colleagues in the INM-3 and INM-4 for valuable support.

The authors declare no competing financial interests.

Correspondence should be addressed to Simone Vossel, Cognitive Neuroscience, Institute for Neuroscience and Medicine (INM-3), Research Centre Juelich, Leo Brandt Str. 5, 52425 Juelich, Germany. E-mail: s.vossel@fz-juelich.de.

DOI:10.1523/JNEUROSCI.3255-16.2017

Copyright $\odot 2017$ the authors $\quad 0270-6474 / 17 / 375334-11 \$ 15.00 / 0$ tions that we make about upcoming events or motor acts. Paradigms in which cues predict the location of a stimulus (Posner et al., 1980) or the motor response to a target (Rushworth et al., 1997) are used to study these effects. Response times (RTs) are accelerated if the cue is valid, whereas slower RTs are observed in invalid trials when the prediction is violated and reorienting of attention or reprogramming of the motor response become necessary. A ventral frontoparietal network including the right temporoparietal junction (TPJ) exhibits enhanced neural activity for spatially invalid (compared with valid) targets (Corbetta et al., 2008). Repetitive transcranial magnetic stimulation applied over the left supramarginal gyrus disrupts the performance in invalidly cued motor (but not spatial) trials (Rushworth et al., 2001a) regardless of the subjects' responding hand or verbal strategies (Rushworth et al., 2001b). 
A

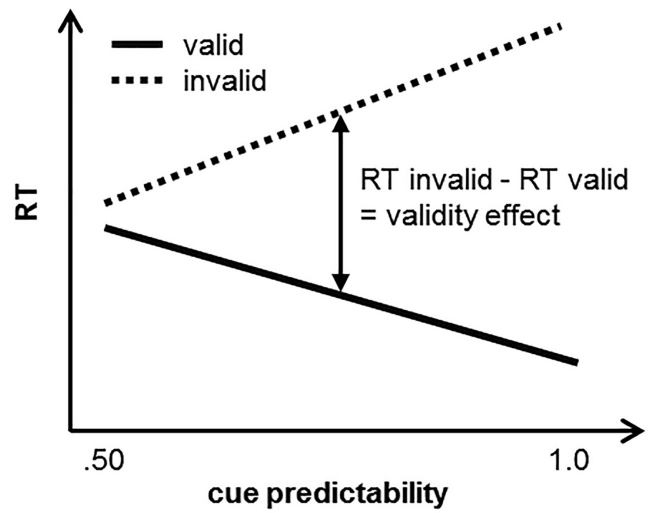

B

Modulation of BOLD responses in valid trials
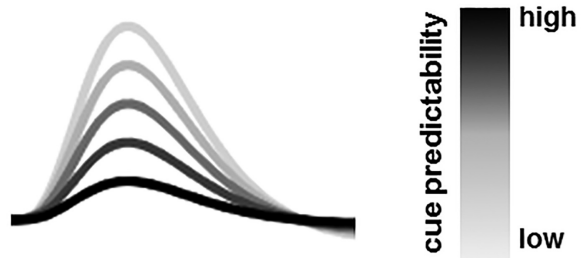

Modulation of BOLD responses in invalid trials

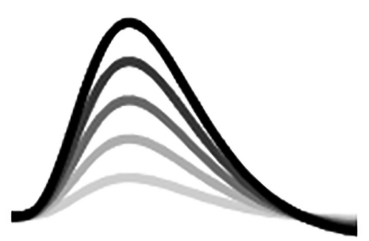

Figure 1. Schematical illustration of the hypothesized effects of cue predictability. A, Illustration of the expected differential effects of cue predictability on RTs in valid and invalid trials. $B$, Computational anatomy that can be inferred from the fMRI results. In predictive coding formulations of hierarchical neuronal processing, precision-weighted prediction errors may be encoded neurally. Belief updating in the hierarchical Gaussian filter model rests on prediction errors in the different levels that are weighted by (level-specific) precision terms. In the present study, we focused on this process at the lower level of the model; that is, on the observation of valid and invalid trials in relation to the expected cue predictability. Here, BOLD amplitudes should be attenuated with higher cue predictability in valid trials, but increase with higher cue predictability in invalid trials (when the prediction is violated). Such effects were identified by including model-derived cue predictability as a parametric modulator for valid and invalid trials in the fMRI analysis and by subsequently contrasting the parametric effects between the two trial types with a planned invalid $>$ valid contrast.

Spatial reorienting is not an all-or-none phenomenon because RTs in valid and invalid trials are affected differentially by the predictability of the cue (i.e., the probability with which the cue correctly predicts the target location in a given trial determined by the proportion of valid and invalid trials in a trial sequence). While RTs in valid trials decrease, RTs in invalid trials increase with higher cue predictability, resulting in bigger validity effects (Fig. 1A).

Moreover, there is evidence that subjects infer the trialwise cue predictability on the basis of past trials and that this estimation can be described by a hierarchical Gaussian filter (a special case of generalized predictive coding or Bayesian filtering) (Mathys et al., 2011; Vossel et al., 2014a). This model estimates hidden states (in our specific case: beliefs about cue predictability and its stability over time) and this estimation can be framed in terms of propagating precision-weighted prediction errors from one hierarchical level to the next. Here, beliefs about cue predictability are represented on the lower level of the model and are influenced by beliefs about the stability (volatility) of cue predictability represented on a higher level and also by subject-specific parameters. Although the higher-level volatility is of major relevance for the flexibility of the model (Behrens et al., 2007; den Ouden et al., 2010), the focus of this study concerned the effects of (lower-level) cue predictability on behavior and BOLD responses. Here, as for RTs, we expected differential effects in valid and invalid trials; that is, increased BOLD responses with higher cue predictability in invalid trials, but decreased BOLD responses with higher predictability in valid trials (Fig. $1 B$ ).

Using a spatial cueing paradigm with saccadic responses, activity patterns as shown in Figure $1 B$ have been observed in the right frontal eye field, TPJ, and putamen (Vossel et al., 2015). However, because saccade preparation is inherently linked to covert shifts of spatial attention (Deubel, 2008), this previous work cannot differentiate between attentional and motor-intentional effects.
In this study, we used two novel cueing tasks to compare directly the effects of cue predictability in the spatial attentional and motor intentional system. The cues either guided spatial attention or signaled the upcoming motor response to the target. The proportion of valid and invalid trials changed unpredictably over time and the subjects' trialwise beliefs about cue predictability were derived from a hierarchical Gaussian filter and were included as parametric regressors for analyzing the BOLD time series. This allowed us to test whether the neural signatures of predictive coding share common mechanisms or if there are different physiological implementations for spatial attention and motor intention. We expected a greater predictability-dependent involvement of left parietal areas in the motor intention task. For spatial attention, we expected a modulation of right TPJ activity (Vossel et al., 2015; Dombert et al., 2016a). In addition, we performed explorative connectivity analyses to test for convergent predictability-dependent coupling patterns in the two functional domains.

\section{Materials and Methods}

Participants. Initially, 34 healthy participants gave written informed consent to take part in the study. Eleven subjects had to be excluded due to head movement $>3 \mathrm{~mm}(n=4)$ or technical problems during scanning $(n=7)$. Therefore, data from 23 subjects ( 13 males, 10 females; age range 18-36; mean age 26 years) were analyzed. All subjects were right-handed, as assessed by the Edinburgh Handedness Inventory (Oldfield, 1971) and had normal or corrected-to-normal vision and no history of neurological or psychiatric disorders. The study was approved by the ethics committee of the German Psychological Society and was performed in accordance with the Code of Ethics of the World Medical Association (Declaration of Helsinki).

Stimuli and experimental paradigm. The tasks were presented on a screen (spatial resolution $1024 \times 768,60 \mathrm{~Hz}$ sampling rate) mounted at the back of the magnet bore. A mirror system attached to the head coil enabled the subjects to see the display at a viewing distance of $240 \mathrm{~cm}$. 
Spatial cues

Motor cues

valid (orient left)

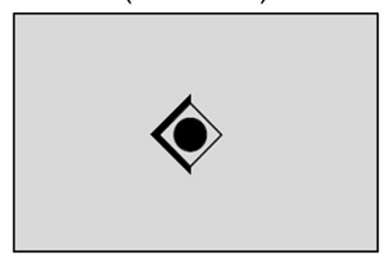

invalid (orient right) valid

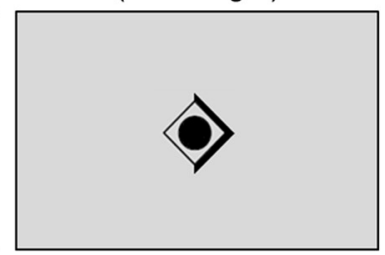

(prepare index finger)

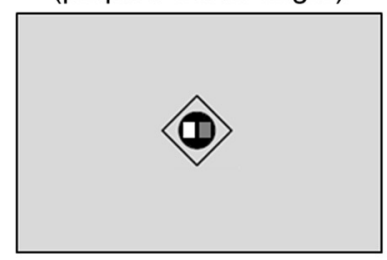

invalid (prepare middle finger)

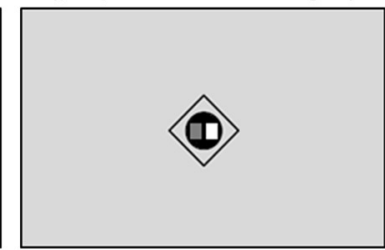

index finger response to upper missing corner

$400 \mathrm{~ms}$

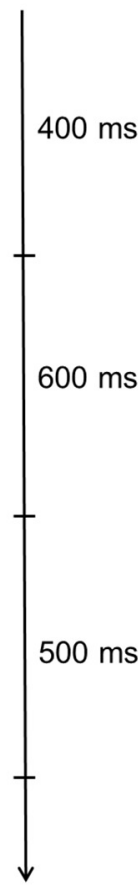

Figure 2. Illustration of the different experimental conditions for the two different tasks. The behaviorally relevant target stimulus was a diamond with a missing corner, which was embedded in a visual search display. The subjects were asked to indicate by button press of one of two adjacent response buttons (for the right index or middle finger) whether the upper or lower corner of the target diamond was missing. The allocation of upper/lower corner to index/middle finger was counterbalanced across subjects. This example shows a configuration in which the subjects should respond with the index finger for diamonds in which the upper corner is missing. The target search display was always preceded by a cue stimulus. In the spatial attention task, this cue indicated the most likely hemifield in which the target would be located (with variable levels of cue predictability). In the motor intention task, the cue indicated the most likely button press response required by the target. This cue consisted of two squares representing the two response buttons. The white button signaled the most likely button press response (with variable levels of cue predictability). Note that the spatial attention task and the motor intention task were presented in different runs.

Participants performed two versions of an adapted cueing paradigm (Egner et al., 2008). They were asked to detect a target stimulus (the diamond with one corner missing) in a visual search display and to indicate by button press whether the upper or lower corner of the target was missing. For their motor response, subjects were provided with a button box with two neighboring response buttons that they pressed with their right index finger or their right middle finger (Fig. 2).

The search display consisted of four diamonds that were positioned in the corners of an imaginary rectangle centered on the fixation diamond $\left(4.8^{\circ}\right.$ eccentric in each visual field; Fig. 2$)$. The different colors of the stimuli in the search display were irrelevant for the present task, but were introduced for reasons of comparability with previous research (Dombert et al., 2016a).

The search display was always preceded by a cue stimulus that was shown for $400 \mathrm{~ms}$. In the spatial attention task, the cue was an arrowhead appearing at the central fixation diamond, indicating the most likely hemifield in which the target would appear (Fig. 2). In the motor intention task, the cue stimulus contained an illustration of the two response buttons for the right index and middle finger (Fig. 2). The white button indicated the most likely button press response required by the target. Therefore, in the example depicted in Figure 2, the subjects prepared a button press with the index finger (valid condition) or a button press with the middle finger (invalid condition). In other words, the target always determined the motor response, but the cue biased the motor preparation toward one or the other response. The response mapping (upper/lower corner missing $\rightarrow$ index/middle finger) was counterbalanced across subjects. Subjects needed to respond to the target within a period of $1500 \mathrm{~ms}$ from target onset. The tasks with motor or spatial cues were presented in two different runs, with counterbalanced order between subjects.
Throughout the experiment, participants were asked to maintain central fixation and to respond as quickly as possible to the target. Moreover, they were instructed to use the cues according to how much they "trusted" them to speed up RTs to the target. The percentage of cue validity $(\% \mathrm{CV})$ changed between levels of $\sim 50 \%, 70 \%$, and $90 \%$ valid trials (cf. Fig. 4B). Participants were not aware of the different levels of $\% \mathrm{CV}$ or when they would change; they were only informed that variations in \%CV would occur over the course of the experiment. In the fMRI experiment, 284 trials were shown in each cueing version. In accordance with standard procedures in computational studies of trialwise inference, target stimuli and trial sequence were identical between the two cueing versions. Each \%CV block consisted of 22 or 32 trials. This block length was chosen to provide participants with sufficient trials to learn the hidden statistical context. Each block contained an equal number of left and right upper and lower targets counterbalanced across valid and invalid cues. Furthermore, 84 null events (only displaying the fixation diamond) were randomly intermixed to jitter trial onsets. Halfway through each version, a 1 min break was introduced by displaying the word "Pause". The total duration of the fMRI experiment (both runs) amounted to 34 min. To familiarize the subjects with the task, fixation, and manual responses, we included a prior practice session in the experiment. This practice took place on the same day or the previous day of the fMRI session and consisted of one run with constant $\% \mathrm{CV}$ of $80 \%$ and one run with changes in \%CV.

Statistical analysis of behavioral data. Classical inference was first performed to investigate whether the different levels of experimentally manipulated cue predictability (\%CV) significantly affected RTs and whether these effects differed for spatial attention and motor intention. Incorrect trials, misses, anticipations, and responses deviating $>2$ SDs from the individual subject's mean RT were excluded from the analysis. 


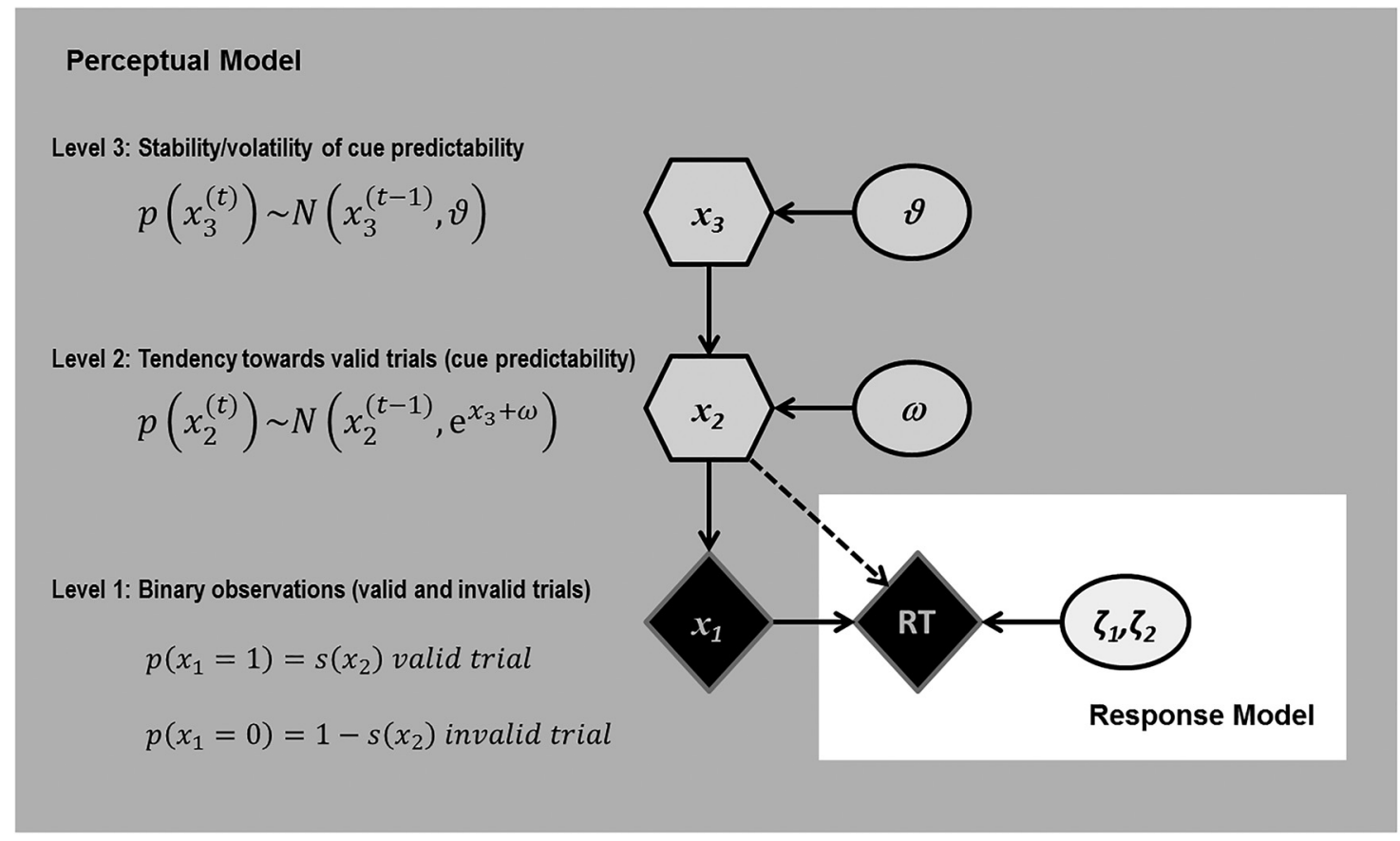

Figure 3. Depiction of the hierarchical Bayesian model (Gaussian filter) for belief updating about cue predictability. The perceptual model (shown on gray background) illustrates the three states $\left(x_{1}, x_{2}, x_{3}\right)$. The higher levels are influenced by constant parameters $\omega$ and $\vartheta$, which affect trialwise changes on the respective level. Circles represent constants and diamonds represent quantities that change over time (trials). Hexagons, like diamonds, represent quantities that change in time but that additionally depend on their previous state in time in a Markovian fashion. The response model parameters $\zeta_{1}$ and $\zeta_{2}$ quantify the intercept and the slope of the linear function of RT and cue predictability $\hat{\mu}_{1}^{(t)}$.

Mean RTs from valid and invalid trials for each subject, \%CV condition and task entered a 2 (validity: valid/invalid) $\times 3(\% \mathrm{CV}: 50 / 70 / 90 \%) \times 2$ (task: spatial attention/motor intention) within-subject ANOVA. Results of this analysis are reported after a Greenhouse-Geisser correction at a significance level of $p<0.05$. Based on evidence for a linear relationship between validity effects (RT invalid - RT valid) and \%CV (Egner et al., 2008; Vossel et al., 2014a; Dombert et al., 2016b; Dombert et al., 2016a), we report linear contrasts for the effects of $\% C V$. We expected to find a significant interaction between validity and $\% \mathrm{CV}$, with decreasing RTs in valid and increasing RTs in invalid trials (i.e., bigger validity effects) with higher \%CV. The same within-subjects ANOVA was performed on accuracy (as the percentage of correct responses).

In a second step, a Bayesian observer model (hierarchical Gaussian filter) was used to estimate trial-by-trial beliefs about cue predictability (i.e., the probability that the cue will be valid) based on the single-trial RTs for each participant (Mathys et al., 2011; Vossel et al., 2014a). The parameters that can be derived from this model also allow for a quantification of individual differences in the trialwise estimation of cue predictability. The model incorporates a perceptual and a response model (Fig. 3). While the perceptual model describes updating of beliefs based on the cue-target outcomes (i.e., observations), the response model is used to derive responses (i.e., RTs) based on these beliefs. Details about the derivation of the equations of the perceptual model are provided in Mathys et al. (2011). In what follows, we describe the model parameters as relevant for the present study.

The perceptual model consists of hierarchically coupled Gaussian random walks that enable a flexible control of updating of the beliefs about cue predictability in each trial in relation to beliefs about volatility and subject-specific parameters. It comprises three states denoted by $x$ (Fig. 3). The state $x_{1}^{(t)}$ represents the environmental state in each trial, which, in the present paradigm, consisted of either a validly or invalidly cued target (with $x_{1}^{(t)}=1$ for valid and $x_{1}^{(t)}=0$ for invalid trials). The probability distribution of the trial being valid (i.e., of $x_{1}^{(t)}=1$ ) is a Bernoulli distribution governed by the next higher state $x_{2}^{(t)}$. Therefore, $x_{2}^{(t)}$ is a single real number that determines the probability of $x_{1}^{(t)}$ being 1 (valid) or 0 (invalid) through a sigmoid (softmax) transformation. $x_{2}^{(t)}$ changes from trial to trial as a Gaussian random walk. Its value depends on the value from the previous trial $t-1$ and the magnitude of its change (i.e., how fast $x_{2}^{(t)}$ changes after new observations) is determined by two quantities: $x_{3}^{(t)}$ (the state of the next upper level of the hierarchy) and a fixed, subjectspecific updating parameter $\omega$. The third state $x_{3}^{(t)}$ represents the belief about the stability of cue predictability and also changes as a Gaussian random walk, with the step size being determined by a second subjectspecific parameter $\vartheta$. The values of the subject-specific parameters $\omega$ and $\vartheta$ are estimated from the individual RT data (see below).

This allowed us to estimate subject-specific beliefs about trial-by-trial variations in probabilistic contingencies. To infer these subject-specific beliefs from the RTs, the perceptual model needs to be inverted; this yields the posterior densities over the three hidden states $x^{(t)}$. In the following, the mean of the subject's posterior belief will be denoted by $\mu^{(t)}$. We use the hat symbol $(\wedge)$ to denote predictions before the observation of $x_{1}^{(t)}$ on a given trial $t$. Therefore, the relevant trialwise quantity for the present study was $\hat{\mu}_{1}^{(t)}$; that is, the posterior belief that the cue will be valid before observation of the outcome of trial $t$ (or, in other words, the estimated probability that the target will appear at the cued location or will require the cued motor response in the upcoming trial). $\hat{\mu}_{1}^{(t)}$ is derived from a sigmoid transformation of $\mu_{2}^{(t-1)}$ as follows:

$$
\hat{\mu}_{1}^{(t)}=s\left(\mu_{2}^{(t-1)}\right)
$$

As described in detail in Mathys et al. (2011), variational model inversion under a mean field approximation yields simple analytical update equations, in which belief updating rests on precision-weighted prediction errors. These update equations provide approximately Bayes-optimal rules for the trial-by-trial updating of the beliefs about $\mu_{2}^{(t)}$ and $\mu_{3}^{(t)}$. Note that this is an individualized approximate Bayes optimality in reference to the subject-specific values for the updating parameters $\omega$ and $\vartheta$.

A response model was used to map the derived posterior beliefs to the observed RTs. In previous work using a saccadic response task with spatial cueing, RTs could most plausibly be explained by the trialwise precision of the prediction at the first level of the perceptual model (Vossel et al., 2014a; Vossel et al., 2014b). However, because we used a novel paradigm with manual responses in this study, we again compared the three alternative response models considered in this previous 
work. Variational Bayesian estimation was used to derive the model parameters based on RTs, as implemented in the HGF toolbox (http:// www.translationalneuromodeling.org/tapas/) running on MATLAB 2012b (The MathWorks). The relative plausibility of the previous response models was compared using a random-effects Bayesian model selection (Stephan et al., 2009). This analysis revealed that the model in which RTs were governed directly by the estimated cue predictability $\hat{\mu}_{1}^{(t)}$ described the data most plausibly. More specifically, this response model describes trialwise RTs as a linear function of the estimated cue predictability $\hat{\mu}_{1}^{(t)}$. The two response model parameters $\zeta_{1}$ and $\zeta_{2}$ parameterize the intercept and the slope of the linear function:

$$
R T^{(t)}= \begin{cases}\zeta_{1 v}-\zeta_{2 v} \hat{\mu}_{1}^{(t)} & \text { for } x_{1}^{(t)}=1 \text { (i.e., valid trial) } \\ \zeta_{1 i}+\zeta_{2 i} \hat{\mu}_{1}^{(t)} & \text { for } x_{1}^{(t)}=0 \text { (i.e., invalid trial) }\end{cases}
$$

Again, like the subject-specific parameters $\omega$ and $\vartheta$ of the perceptual model, these response model parameters were estimated for each subject from the individual RT data.

Eye movement recording and analysis. Eye movement data were acquired using an EyeLink 1000 MR-compatible eye-tracker system (SR Research) at a sampling rate of $500 \mathrm{~Hz}$. Before the task, a 9 or 5 point calibration was performed, followed by a validation to ensure that errors were $<1^{\circ}$. Data were processed using the ILAB toolbox (Gitelman, 2002) in MATLAB (The MathWorks). The amount of time spent within a fixation zone of $1.5^{\circ}$ from the central fixation point was analyzed for the time between cue and target appearance, as well as for the time period between target and response. Percentage of fixation time within the central ROI in the cue-target period was compared between spatial and motor cues with a paired $t$ test. Fixation between target appearance and response was analyzed with a 2 (task: spatial attention/motor intention) $\times 2$ (validity: valid/invalid) within-subject ANOVA.

MRI data acquisition. Using a 3 T MRI system (Trio; Siemens), T2*weighted EPI images with BOLD contrast were acquired with a repetition time of $2.2 \mathrm{~s}$ and an echo time of $30 \mathrm{~ms}$. Two functional runs were acquired consisting of $462 \mathrm{EPI}$ volumes for each run (i.e., for each task). Each volume consisted of 36 axial slices with interleaved slice acquisition. The field of view was $200 \mathrm{~mm}$ using a $64 \times 64$ image matrix, which resulted in a voxel size of $3.1 \times 3.1 \times 3.0 \mathrm{~mm}^{3}$. The first five volumes were discarded from the analysis to allow for $\mathrm{T} 1$ equilibration effects. The remaining $2 \times 457$ volumes were analyzed using the Statistical Parametric Mapping software SPM12 (Wellcome Department of Imaging Neuroscience, London; Friston et al., 1995; http://www.fil.ion.ucl.ac.uk/ $\mathrm{spm})$. Images were bias-corrected. Slice acquisition time differences were corrected using sinc interpolation to the middle slice. During spatial realignment, a mean EPI image was computed for each subject and spatially normalized to the MNI template using the segmentation function. Subsequently, the resulting transformation was applied to the individual EPI volumes to translate the images into standard MNI space and resample them into $2 \times 2 \times 2 \mathrm{~mm}^{3}$ voxels. Finally, the normalized images were spatially smoothed using an $8 \mathrm{~mm}$ full-width half-maximum Gaussian kernel.

Statistical analysis of imaging data. At the single-subject level, the spatial attention and motor intention tasks were included as separate sessions in a general linear model of the BOLD responses. For each session, regressors of interest for left and right valid and invalid trials were defined. The resulting stimulus functions were convolved with a canonical hemodynamic response function (HRF) and its first (temporal) derivative. For each task regressor, cue predictability $\hat{\mu}_{1}^{(t)}$ as derived from the single-subject computational modeling was included as a parametric modulator. Note that parametric modulations in SPM are used to test whether the trialwise amplitude of the BOLD response in an experimental condition varies with a continuous variable of interest. RTs were added as a second (orthogonalized) parametric regressor to capture residual variability that was not explained by cue predictability $\hat{\mu}_{1}^{(t)}$. Error trials (anticipations, misses, and incorrect responses) and outliers (RTs above or below 2 SDs from the subject's mean) were discarded from the effects of interest and modeled separately. In addition, the rest period and 12 movement parameters of the (rigid body) realignment (six motion parameters and their power of two; Friston et al., 1996) were included in the design matrices as nuisance regressors. Data were high-pass filtered at $1 / 128 \mathrm{~Hz}$.

As shown in Figure 1, the aim of the current study was to identify and compare brain areas in which BOLD responses are modulated differentially by cue predictability in valid and invalid trials, with potentially negative parametric effects for valid trials (i.e., smaller BOLD responses with higher predictability) and positive parametric effects for invalid trials (i.e., higher BOLD responses with higher cue predictability). For this reason, we focused on planned comparisons of the parametric regressors of invalid and valid trials ( $t$-contrasts of invalid $>$ valid) in each task and compared these between the two different tasks using interaction contrasts. These interaction contrasts between validity (valid/invalid) and task (spatial attention/motor intention) thus isolate domain-specific correlates of predictive processing in one or the other task.

Because no significant interaction with hemifield was observed in the analysis of probability-independent effects (contrast of invalid $>$ valid trials for the HRF regressors) (data not shown) and to increase the trial numbers for the parametric modulation effects, the analysis of cue predictability was based on a first-level design matrix with only two regressors for all valid and all invalid trials in each task, respectively. At the group level, the first-level contrast images for variations of BOLD amplitudes with cue predictability $\hat{\mu}_{1}^{(t)}$ were analyzed with a 2 (task: spatial attention/motor intention) $\times 2$ (validity: valid/invalid) within-subject random-effects ANOVA. As explained above, differential predictabilitydependent effects for spatial attention and motor intention were investigated with interaction contrasts (spatial attention [invalid $>$ valid] $>$ motor intention [invalid $>$ valid]; motor intention [invalid $>$ valid] $>$ spatial attention [invalid $>$ valid]). To ensure that the interaction effects were indeed due to significantly higher effects in invalid as in valid trials in the respective task version (and not only due to a reversed effect in the other task), the interaction contrasts were inclusively masked by the invalid $>$ valid contrast of one task with a mask threshold of $p<0.01$ (uncorrected). We also tested for a common predictability-dependent effects in the two cueing tasks using a conjunction analysis of the two invalid $>$ valid contrasts for the two tasks (SPM conjunction null hypothesis: spatial attention [invalid $>$ valid] $\cap$ motor intention [invalid $>$ valid]). All reported activations were significant at $p<0.05$ familywise error (FWE), corrected at the cluster level with a voxel-level cutoff of $p<$ 0.001 . Because we had a strong a priori hypothesis that the right TPJ would exhibit predictability-dependent effects (Vossel et al., 2015; Dombert et al., 2016a), we used small volume correction in the spatial attention [invalid $>$ valid] $>$ motor intention [invalid $>$ valid] contrast using a $12 \mathrm{~mm}$ sphere centered upon the coordinates from Vossel et al. (2015) [MNI coordinates $(46-466)]$. Results from this ROI analysis are reported at a significance level of $p<0.05 \mathrm{FWE}$, corrected for the search volume. Brain regions were defined anatomically using the SPM Anatomy Toolbox (Eickhoff et al., 2005) for those regions that have been cytoarchitectonically mapped and the Automated Anatomical Labeling atlas (Tzourio-Mazoyer et al., 2002) for the remaining regions.

Psychophysiological interaction (PPI) analyses. Because no common activation patterns were revealed by the conjunction analysis of probabilitydependent effects in the two domains (i.e., spatial attention and motor intention), we used PPI analyses (Friston et al., 1997) to test for potentially converging cue-predictability-dependent connectivity patterns of the different seed regions derived from the former analysis [right TPJ for spatial attention; left angular gyrus (ANG) and anterior cingulate cortex (ACC) for motor intention]. PPIs explain the responses in cortical areas in terms of an interaction between the influence of another area and an experimental manipulation. This allows for a whole-brain analysis of context-dependent coupling with one predefined seed region.

For each seed region, time series were extracted from the nearest local maximum within a radius of $8 \mathrm{~mm}$ from the group maxima. The first eigenvariate was then computed across all suprathreshold voxels within 4 $\mathrm{mm}$ of the subject-specific maxima. The resulting BOLD time series were adjusted for effects of no interest (e.g., rest periods, error trials, and movement parameters) and deconvolved to generate time series of the neuronal signal. These time series were used to construct the first-level design matrices for the PPIs. 
Table 1. Behavioral data

\begin{tabular}{lllll}
\hline & & $\% \mathrm{CV}$ & \\
\cline { 4 - 5 } Task & Validity & $50 \%$ & $70 \%$ & $90 \%$ \\
\hline Mean RTs, ms ( $\left( \pm\right.$ SEM) $^{a}$ & & & & \\
Spatial attention & Valid & $628.1( \pm 22.6)$ & $629.6( \pm 21.8)$ & $626.1( \pm 21.9)$ \\
& Invalid & $651.3( \pm 20.7)$ & $657.8( \pm 24.5)$ & $672.6( \pm 24.7)$ \\
Motor intention & Valid & $618.2( \pm 19.1)$ & $622.7( \pm 18.8)$ & $611.7( \pm 18.5)$ \\
& Invalid & $646.2( \pm 19.1)$ & $653.6( \pm 21.7)$ & $662.4( \pm 20.4)$ \\
Mean accuracy, \% correct & & & & \\
responses ( \pm SEM) & $b$ & & & \\
Spatial attention & Valid & $91.2( \pm 1.2)$ & $87.6( \pm 1.7)$ & $88.7( \pm 0.9)$ \\
& Invalid & $85.0( \pm 2.0)$ & $85.5( \pm 2.0)$ & $84.0( \pm 2.0)$ \\
Motor intention & Valid & $89.6( \pm 1.4)$ & $87.6( \pm 1.7)$ & $89.5( \pm 1.2)$ \\
& Invalid & $85.5( \pm 1.3)$ & $83.6( \pm 1.7)$ & $83.4( \pm 1.8)$ \\
\hline
\end{tabular}

${ }^{a}$ Mean RTs for spatial attention and motor intention separately for valid and invalid trials in the three different \%CV levels.

${ }^{b}$ Mean accuracy in the different experimental tasks and conditions.

Our PPI analysis was, necessarily, more complicated than a standard PPI analysis. This is because a standard PPI analysis tests for a single interaction between a physiological variable and a psychological variable. However, in our case, the psychological variable of interest is itself an interaction: an interaction between validity and predictability. This means that we were effectively testing for a three-way interaction between a physiological and two psychological variables. In turn, this required us to model, not only the main effects of the psychological and physiological variables, but also two-way interactions between the physiological variable and the psychological variables validity (valid/invalid) and model-derived cue predictability. The resulting explanatory variables therefore contain multiple PPI terms, rendering it a generalized PPI. Analyses were performed with the Generalized PPI (gPPI) Toolbox (McLaren et al., 2012).

In detail, the design matrices for the three separate PPI analyses at the single-subject level contained nine experimental regressors: four regressors for valid and invalid trials and their parametric modulation by predictability; four PPI regressors for the interactions between the physiological variable (i.e., the time series of the seed region) and valid trials, invalid trials, and their parametric modulation by predictability; and one regressor for the physiological variable. Model estimation was performed and first-level contrast images were created for the PPI regressor of the parametric modulator (cue predictability $\hat{\mu}_{1}^{(t)}$ ) in valid and invalid trials.

At the second (i.e., group) level, the first-level contrast images entered a conjunction analysis of the invalid $>$ valid contrast of each of the three seed regions (right TPJ for spatial attention, left ANG and ACC for motor intention). The "intermediate null hypothesis" in SPM was chosen to test for a common effect in two or more contrasts. Again, reported activations were significant at $p<0.05$ FWE, corrected at the cluster level with a voxel-level cutoff of $p<0.001$. This analysis identified areas where the connectivity with two or more regions decreased with higher estimated cue predictability in valid trials and in which connectivity increased with higher estimated cue predictability in invalid trials.

\section{Results}

\section{Behavioral data}

Table 1 provides an overview of mean RTs and accuracy in the different experimental conditions. Figure $4 A$ depicts mean validity effects (RT invalid - RT valid) for both tasks in the three experimentally manipulated \%CV levels.

The 2 (task: spatial attention/motor intention) $\times 2$ (validity: valid/invalid) $\times 3$ (\%CV: 50/70/90) within-subject ANOVA on individual mean RTs yielded a significant main effect of validity $\left(F_{(1,22)}=27.56, p<0.001\right)$, reflecting generally slower responses in invalid compared with valid trials. As expected, the validity $X$ $\% \mathrm{CV}$ interaction effect was significant (linear trend: $F_{(1,22)}=$ 13.77, $p=0.001$ ), indicating higher differences between invalid and valid trials with higher \%CV (Fig. 4A). There were no general differences in RTs between both task versions (nonsignificant main effect of task: $\left.F_{(1,22)}=1.45, p=0.24\right)$. The same results were observed when task order was included as a between-subject factor in the above ANOVA and task order did not interact with any of the effects.

The same ANOVA was performed on percentage accuracy, revealing only a main effect of validity $\left(F_{(1,22)}=14.36, p=\right.$ $0.001)$. Subjects were more accurate in valid than in invalid trials. Again, this effect was not influenced by the order of task administration, nor did it interact with any of the other factors.

In a next step, trialwise estimates of cue predictability $\hat{\mu}_{1}^{(t)}$ were derived from the hierarchical Gaussian filter. These estimates are governed by the constant subject-specific parameters determining the step size of the random walks at the second $(\omega)$ and third level $(\vartheta)$. Comparing these parameters between both task versions revealed no significant differences in any of the parameters. In addition, the response model parameters $\zeta_{1 v}, \zeta_{1 i}, \zeta_{2 v}$, and $\zeta_{2 i}$, quantifying the absolute level of RTs and the strength of the dependency on $\hat{\mu}_{1}^{(t)}$ did not differ significantly between the two task versions. There were no significant correlations between the model parameters from the spatial attention and motor intention tasks, over subjects. Figure $4 B$ depicts $\hat{\mu}_{1}^{(t)}$ calculated on the basis of the mean model parameters of the whole group. Figure $4 C$ shows observed valid and invalid RTs in relation to predicted RTs for different values of model-derived cue predictability $\hat{\mu}_{1}^{(t)}$ (binned in 0.1 steps). A $2 \times 4 \times 2$ within-subject ANOVA with the factors validity (valid/invalid), cue predictability $\hat{\mu}_{1}^{(t)}(>0.55 /$ $>0.65 />0.75 />0.85)$ and task (spatial attention/motor intention) revealed a main effect of validity $\left(F_{(1,22)}=33.03, p<0.001\right)$ and, as expected, a significant validity $\times$ cue predictability $\hat{\mu}_{1}^{(t)}$ interaction (linear contrast: $F_{(1,22)}=17.63, p<0.001$ ), showing that the differences between RTs in invalid and valid trials increased with higher values of $\hat{\mu}_{1}^{(t)}$. This was due to decreasing RTs in valid and increasing RTs in invalid trials.

In sum, these analyses showed that RTs in the two cueing paradigms followed the hypotheses from the predictive coding framework. Moreover, analyses revealed that the dynamics of the estimation of cue predictability were comparable between the two tasks because there were no significant differences in the subject-specific parameters of the hierarchical Gaussian filter.

\section{Eye movement data}

Data from five subjects had to be discarded due to poor tracking inside the MR scanner. Nonetheless, eye movements were monitored visually in these subjects on the camera screen. The remaining subjects fixated in (mean \pm SEM) $97.7 \pm 0.45 \%$ of the motor and $97.5 \pm 0.59 \%$ of the spatial cueing task during the cue-target period. Fixation performance did not differ between the two tasks $\left(t_{(17)}=0.73, p=0.47\right)$. The 2 task (spatial attention/ motor intention) $\times 2$ validity (valid/invalid) within-subject ANOVA on the percentage fixation between target and response showed no significant effect of task $\left(F_{(1,17)}=0.3, p=0.59\right)$ or validity $\left(F_{(1,17)}=0.02, p=0.89\right)$ and no interaction of the two factors $\left(F_{(1,17)}=0.002, p=0.96\right)$.

\section{fMRI data}

The purpose of this study was to compare the differential parametric modulation by cue predictability in invalid and valid trials between the two tasks (in analogy to the differential behavioral effects of cue predictability in valid and invalid trials). Common activity patterns should be revealed by a conjunction analysis (spatial attention [invalid $>$ valid] $\cap$ motor intention [invalid $>$ 
A

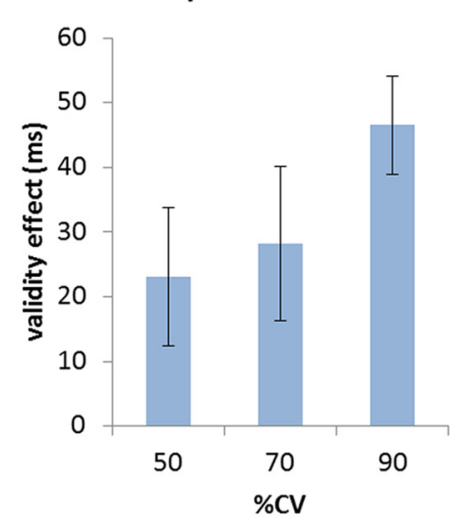

B

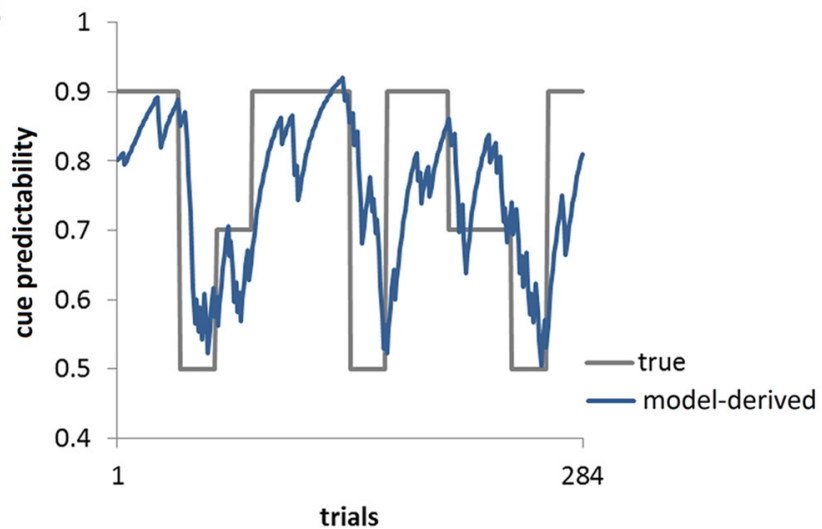

C

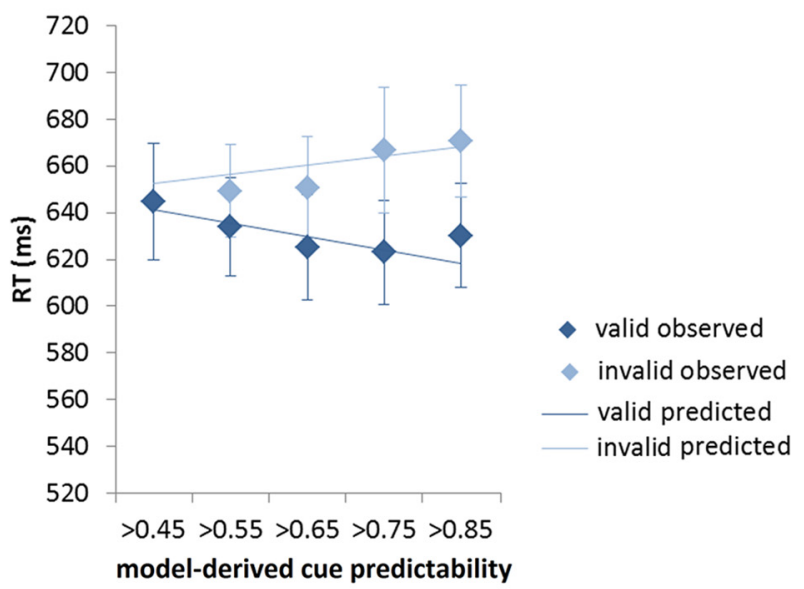

Motor intention
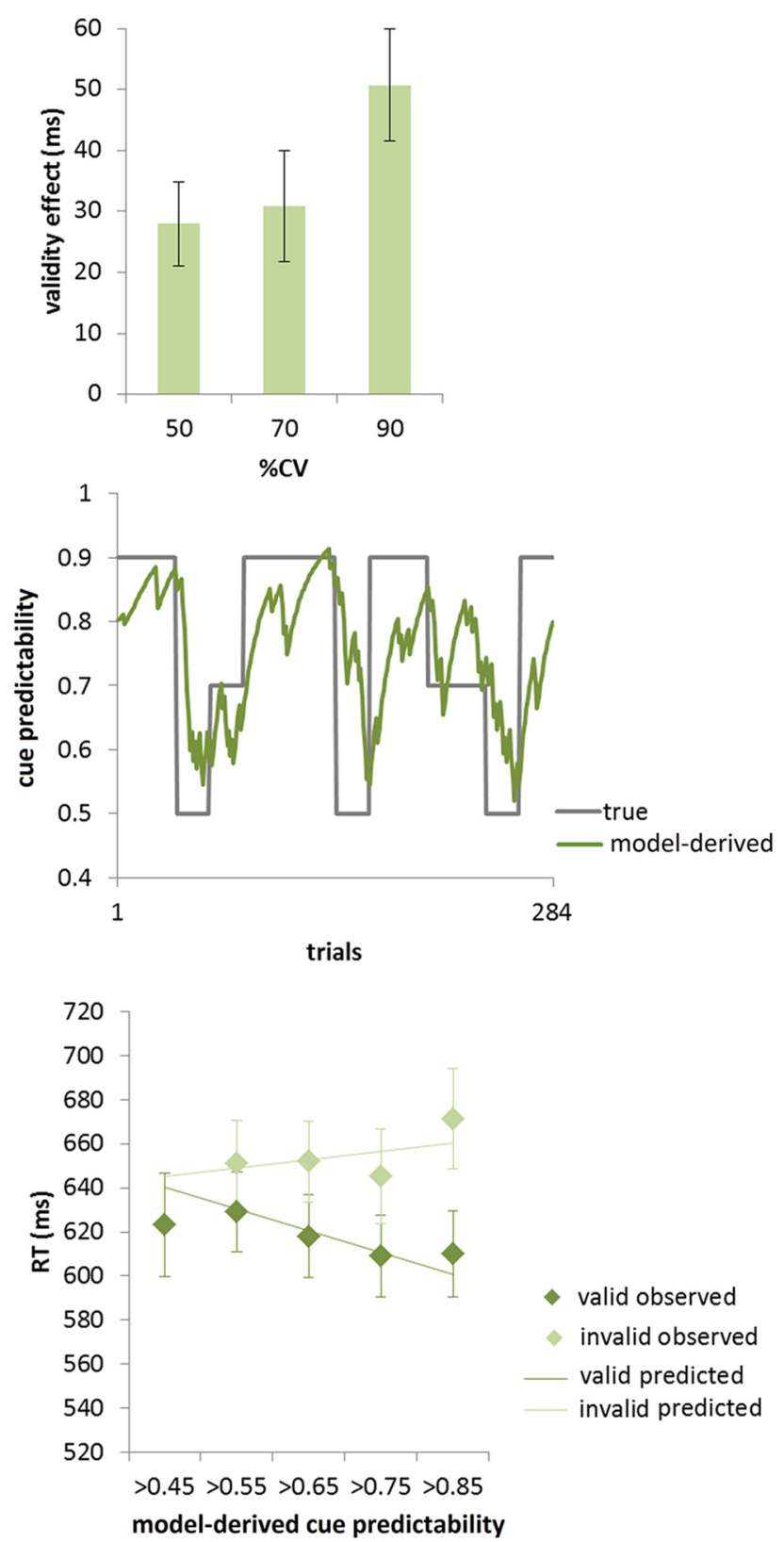

Figure 4. Behavioral data. A, Validity effects (RT invalid - RT valid) for each \%CV block in each task. Error bars indicate SEM. B, Trial-by-trial changes in cue predictability $\hat{\mu}_{1}^{(t)}$ (i.e., the subjects' belief that the cue will be valid) in relation to the experimentally manipulated \%CV over the 284 trials for the spatial attention and motor intention task, respectively. For this graph, $\hat{\mu}_{1}^{(t)}$ was calculated on the basis of group average values of the model parameters, which did not differ between the two tasks. Note, however, that the profiles look different for each individual subject in each task and that individual cue predictability values entered the fMRI analyses. C, Observed and predicted RTs in valid and invalid trials as a function of the trial-by-trial estimate of cue predictability $\hat{\mu}_{1}^{(t)}$ for both task versions (calculated on the basis of group average values of the model parameters). Error bars indicate SEM. Note that there was an insufficient number of trials for the invalid condition in the lowest probability bin, so this data point is missing.

valid]). However, this analysis did not yield any significant results, suggesting that there was no detectable overlap in the brain areas mediating predictability-dependent processing in the spatial attention and motor intention task.

Differential (i.e., domain-specific) activity patterns should be reflected in the two interaction contrasts: spatial attention [invalid $>$ valid] $>$ motor intention [invalid $>$ valid]; motor intention [invalid $>$ valid] $>$ spatial attention [invalid $>$ valid]; each masked with the simple contrast of invalid $>$ valid in the respective task. The first interaction contrast revealed that there were no whole-brain results reaching cluster-level significance for the spatial attention $>$ motor intention effect. However, a significant effect was obtained in a ROI analysis of the right TPJ with a sphere centered on the coordinates from Vossel et al. (2015) ( $p_{\mathrm{FWE}}$-corrected for the search volume) $(x=52, y=-56, z=8 ; 10$ voxel; $t=3.7)$. In this region, there was a positive parametric modulation effect for invalid trials, with parameter estimates around zero for valid trials (Fig. 5A). For the reverse interaction contrast, we found differential parametric modulation effects for invalid versus valid trials in the left ANG $(x=-38, y=-58, y=42 ; 411$ voxel; $t=5.22)$ and the left ACC $(x=-8, y=36, z=24 ; 156$ voxel; $t=4.92)$, which were stronger in the motor intention than in the spatial attention 


\section{A \\ invalid > valid: spatial attention > motor intention}
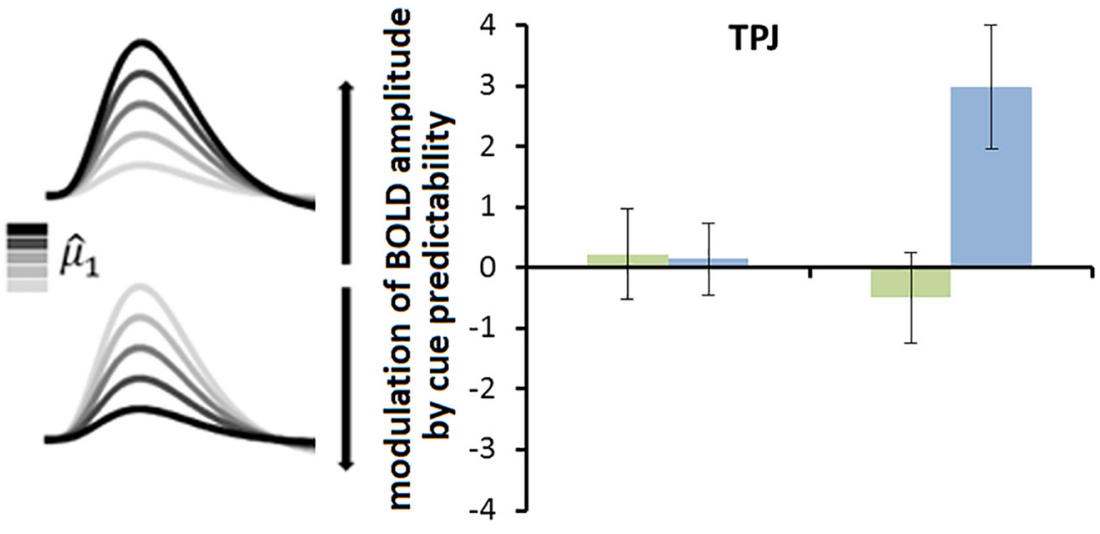

valid

invalid

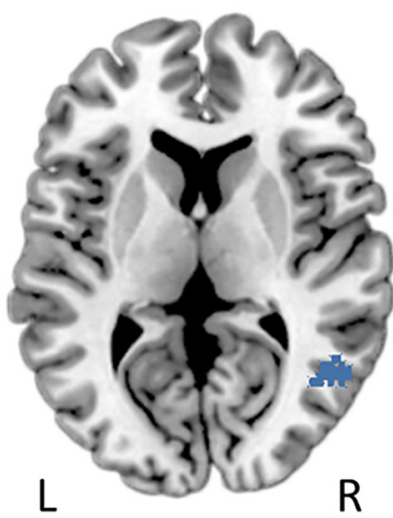

B

invalid > valid: motor intention > spatial attention
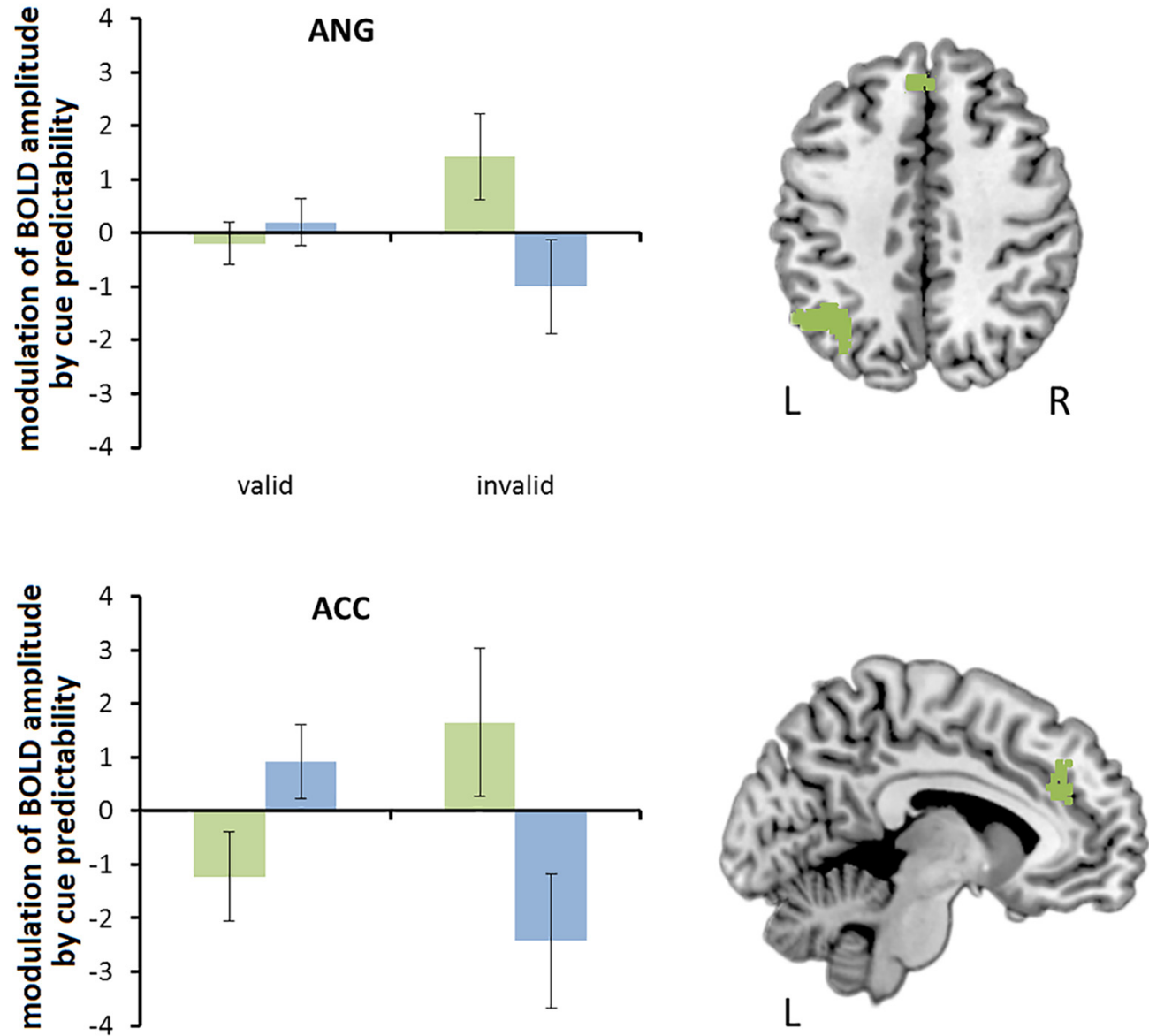

valid

invalid

Figure 5. fMRI data: differential cue-predictability-dependent effects for spatial attention and motor intention. $A$, Results of the first planned interaction contrast (spatial attention [invalid $>$ valid] $>$ motor intention [invalid $>$ valid]): increasing BOLD responses in invalid trials and unmodulated BOLD responses in valid trials with higher values of model-derived cue predictability $\hat{\mu}_{1}^{(t)}$ in the right TPJ in the spatial attention task (blue) compared with the motor intention task (green). $\boldsymbol{B}$, Results of the second planned interaction contrast (motor intention [invalid $>$ valid] $>$ spatial attention [invalid $>$ valid]): increasing BOLD responses in invalid trials and decreased or unmodulated BOLD responses with higher values of model-derived cue predictability $\hat{\mu}_{1}^{(t)}$ in valid trials in the left ANG and left ACC in the motor intention task (green) compared with the spatial attention task (blue). L, Left hemisphere; $R$, right hemisphere. 

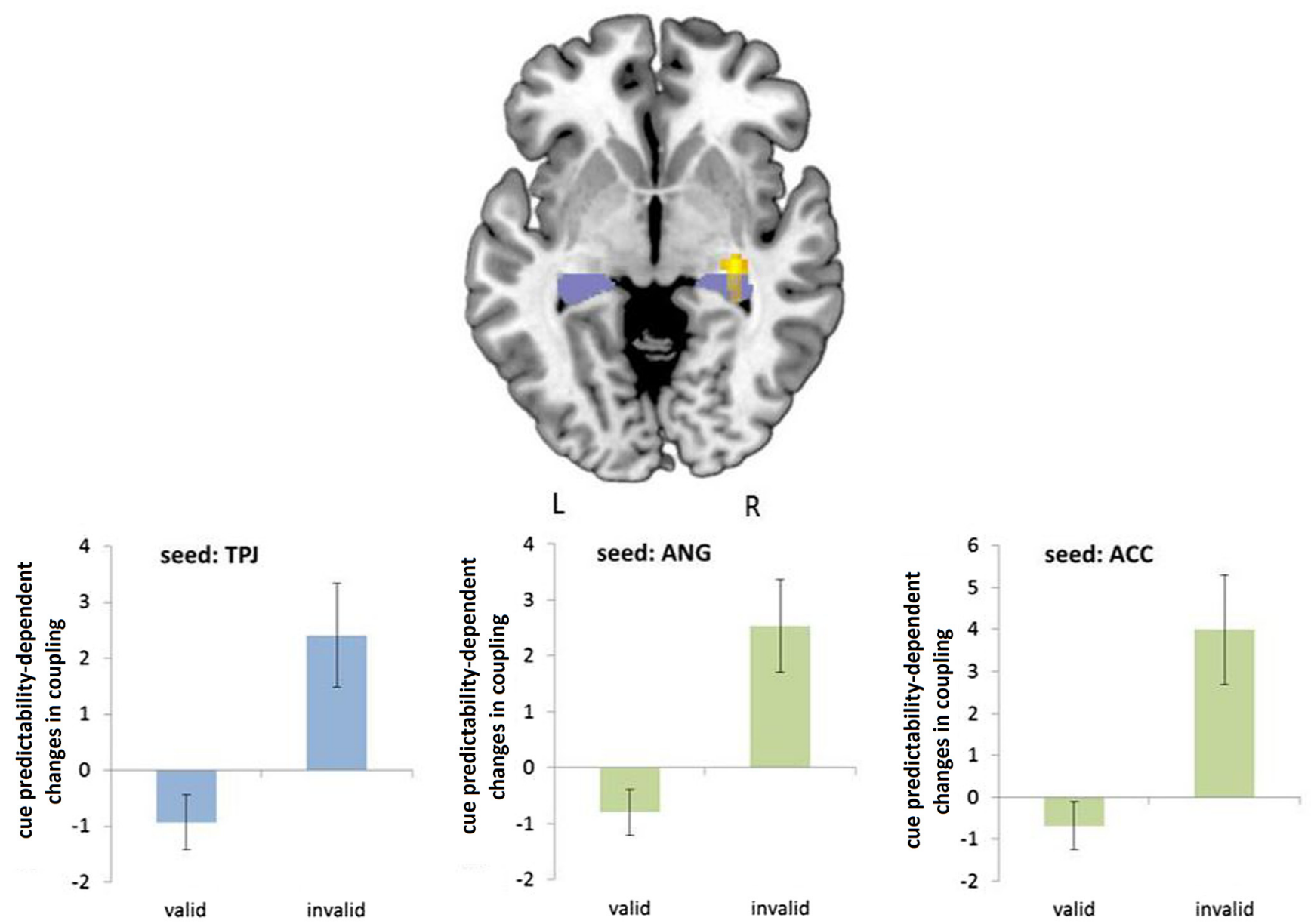

Figure 6. Results of the conjunction analysis of cue-predictability-dependent coupling changes with the right TPJ during spatial attention and the left ANG and ACC during motor intention (yellow). The hippocampus (violet) is shown according to the Automated Anatomical Labeling atlas provided in MRIcroN. Analysis of the parameter estimates for the parametric PPI regressors in valid and invalid trials revealed that a significant predictability-dependent coupling effect with the hippocampus was present for all three seed regions. Blue bars indicate the spatial attention task; green bars, the motor intention task. L, Left hemisphere; R, right hemisphere.

task. The parameter estimates for the parametric regressor cue predictability $\hat{\mu}_{1}^{(t)}$ were positive for invalid trials, reflecting an increased response with higher estimated probability that the cue would be valid (Fig. 5B). In contrast, parameter estimates were zero or negative in valid trials, reflecting no or a decreasing modulation with higher estimated cue predictability.

\section{PPI results}

We used PPI analyses to investigate whether there was a common brain region exhibiting predictability-dependent coupling changes with right TPJ during spatial attention and left ANG or ACC during motor intention, respectively. Figure 6 shows the results of the conjunction analysis testing for significant PPI effects in at least two of the three contrasts capturing the effects of validityand predictability-dependent coupling with the three seeds. The only region in which a significant effect at the cluster level could be observed was located in the anterior part of the right hippocampus ( $x=30, y=-24, z=-4 ; 131$ voxels; $t=2.85)$. Post hoc analyses of the $\beta$ estimates of the parametric PPI regressors revealed that a significant difference between cue-predictabilitydependent coupling in valid and invalid trials was present for all three seed regions (two-sided paired $t$ tests: TPJ seed: $t_{(22)}=$ $-3.4, p=0.003$; ANG seed: $t_{(22)}=-3.8, p=0.001$; ACC seed: $t_{(22)}=-3.5, p=0.002$; see bar charts in Fig. 6).

\section{Discussion}

The present study combined computational modeling of behavior with fMRI to characterize common and distinct cortical mechanisms for predictive processing in spatial attentional and motor intentional systems. Manual RTs in two probabilistic cueing tasks were similarly affected by unsignaled changes in the predictability of a cue that indicated either the location of or the required response to a target stimulus. Differential parametric effects of BOLD responses by model-derived cue predictability in valid and invalid trials were regarded as signatures of the belief updating by precision-weighted prediction errors. Brain areas exhibiting such activity patterns were distinct for spatial attention and motor intention, with an involvement of the left ANG and ACC for motor intention and the right TPJ for spatial attention. In these areas, BOLD amplitudes were increased in invalid trials with higher estimated cue predictability (i.e., when an invalidly cued target was more unexpected) and decreased (ACC) or unmodulated (TPJ and ANG) in valid trials. There were no common areas in which cue predictability modulated neural activity in both domains. However, connectivity analyses revealed that the right hippocampus contributed to predictive processing in all three areas (TPJ during spatial attention; ANG and ACC during motor intention). Our results therefore suggest that, although the 
flexible control by inferred cue predictability recruited differential cortical structures in the two cognitive domains (i.e., spatial attention, motor intention), these structures all showed predictabilitydependent coupling with the hippocampus.

\section{Behavior}

In both the spatial attention and the motor intention task, RTs were equally affected by invalid cue information, as well as by experimentally manipulated changes in the probability that the cue would be valid. Moreover, there were no differences in the overall level of RTs or accuracy. Similarly, there were no significant differences in the parameters of the hierarchical Gaussian filter governing the trialwise estimation of cue predictability between the two tasks. This is consistent with previous studies showing the sensitivity of behavioral responses to probabilistic context during spatial attention (Vossel et al., 2014a) and motor intention (Bestmann et al., 2008). However, the model parameters were not correlated between the spatial attention and motor intention task. This contrasts with findings from the comparison of a spatial and a feature-based attention task, where the updating parameter of the second level of the model $(\omega)$ was significantly correlated between the two task versions (Dombert et al., 2016a). Consistent with these behavioral results, Dombert et al. (2016a) found a common modulation of reorienting-related activity in the left intraparietal sulcus, whereas the conjunction analysis in the present study did not reveal any significant common effect.

\section{Distinct and common mechanisms of cue-predictability- dependent processing}

Differential effects mediated (in our predictive processing model) by precision-weighted prediction errors were found in the left ANG and ACC for motor intention and in the right TPJ for spatial attention. Here, BOLD amplitudes increased with higher model-derived cue predictability in invalid trials. In TPJ and ANG, there was no substantial modulatory effect in valid trials. Although a negative parametric modulation effect would have been predicted from theoretical grounds, this finding is consistent with data from Lasaponara et al. (2011), who showed that effects of higher percentage of cue validity on RTs and ERPs can be stronger for cueing costs (invalid trials compared with neutral trials) than for benefits (valid trials compared with neutral trials).

The effect in the right TPJ replicates findings from previous studies using the same modeling approach with either saccadic responses (Vossel et al., 2015) or manual responses (Dombert et al., 2016a). It also extends these previous findings by showing that this effect was specific for the spatial attention condition because it was not observed for the motor intention task (cf. Fig. 5A). Because we were able to differentiate between attentional and motor intentional aspects in the present paradigm, we can ascribe the effect in TPJ to purely spatial attentional mechanisms. In other words, the preparation of eye movements or the allocation of covert spatial attention seem to involve different mechanisms than the preparation of limb movements.

Conversely, reorienting after invalid motor (but not spatial) cues was affected by model-derived cue predictability in the left ANG and the ACC extending into the left medial superior frontal gyrus. The finding that cue-predictability-dependent effects in a feature-based cueing task without a motor intentional component were observed in yet another brain region in the study by Dombert et al. (2016a) supports the interpretation that the effects in the present study were driven by the motor intentional component rather than by the expectation of a specific target feature. The area in the ACC has also been described as the rostral cingu- late zone (RCZ). Our findings are consistent with a study on reward learning of actions, which reported that the RCZ responded stronger to negative events in a probabilistic reversal learning task when the reward rate was high (Jocham et al., 2009). Similarly, in our study, the BOLD response in this region to invalidly cued motor responses was increased with higher estimated cue predictability even though there was no reward associated with the action. The ACC has also been shown to be especially triggered during cognitively demanding actions after context-specific behavioral surprise (Tobia et al., 2016), as well as during action selection when different response alternatives are competing (Botvinick et al., 1999), which would correspond to invalid trials in the present task. Similarly, predictability-dependent modulation of ACC activity during the motor intention task could also reflect the suppression of the previously prepared motor response. van Gaal and colleagues (2010) characterized a neural network comprising the ACC responsible for the inhibition of responses during a go/no-go task. Our results support the previous associations of ACC with response conflict and selection, but add that this area is modulated by the inferred cue predictability regardless of external reward.

According to probabilistic fiber tracking, the ACC is interconnected structurally with a subregion of the ANG (Caspers et al., 2011), which also showed predictability-dependent effects in the motor intention task. It has to be noted that this parietal activation was located more posteriorly than the activation reported by previous studies on motor intention in the supramarginal gyrus, which did not consider predictability-dependent effects (Rushworth et al., 2001b). Ranganath and Ritchey (2012) suggest that the ANG might be a candidate area for integrating contextual information due to its connection to the hippocampus and a widespread posterior medial system. Another view is that the ANG is a core region for providing an interface between the converging bottom-up multisensory inputs and the top-down predictions in the perception-to-action loop and that this can explain its involvement in a variety of functions (Seghier, 2013). Our data extend these previous findings by showing that activity in the ANG is modulated by probabilistic context on a short trial-bytrial time scale and that this effect is specific for situations with a motor intentional component.

The conjunction analysis of predictability-dependent processing did not detect any common brain regions for spatial attention and motor intention. This finding, together with the significant differential effects reflected in the interactions of task and validity discussed above, argue against a mere "frequency detector" module in the brain, which simply responds to rare events. Interestingly, however, the additional PPI analysis revealed a converging coupling pattern between all three seed regions (TPJ, ACC, and ANG) and the anterior hippocampus for predictability-dependent processing. The hippocampus has been shown to encode the predictability or expected uncertainty (entropy) in choice and sequential RT tasks (Strange et al., 2005; Harrison et al., 2006). Moreover, a recent study has shown that update signals in different brain structures lead to activity changes in the hippocampus and other medial temporal lobe structures, which may suggest that these latter regions provide an online store or neural representation of a current internal model (Boorman et al., 2016). Our data, together with these previous results, provide evidence that the trialwise inference of predictability (involving the temporal integration and processing of relationships between events) recruits the hippocampal system regardless of the content of the encoded information (motoric or spatial). Therefore, the hippocampal system can process different 
types of (spatial and nonspatial) stimulus information flexibly (Viard et al., 2011) and support the formation of internal models to control perception and action in an uncertain world.

In conclusion, our findings provide novel insights into the generality and specificity of the computational anatomy underlying the flexible control of attention and intention in the human brain. Our data, together with previous findings in the attentional domain, argue for the notion that precision-weighted prediction errors induce belief updating in each cognitive domain separately, but that the necessity to relate new information to previous events and integrating it into memory involves crosstalk with the hippocampus in both systems regardless of the informational content. Therefore, our results confirm the previously reported dissociation and complementary lateralization of spatial attention and motor intention in the parietal cortex. At the same time, they highlight similarities of both systems in terms of computational principles and connectivity profiles.

\section{References}

Behrens TE, Woolrich MW, Walton ME, Rushworth MF (2007) Learning the value of information in an uncertain world. Nat Neurosci 10:12141221. CrossRef Medline

Bestmann S, Harrison LM, Blankenburg F, Mars RB, Haggard P, Friston KJ, Rothwell JC (2008) Influence of uncertainty and surprise on human corticospinal excitability during preparation for action. Curr Biol 18:775780. CrossRef Medline

Boorman ED, Rajendran VG, O'Reilly JX, Behrens TE (2016) Two anatomically and computationally distinct learning signals predict changes to stimulus-outcome associations in hippocampus. Neuron 89:1343-1354. CrossRef Medline

Botvinick M, Nystrom LE, Fissell K, Carter CS, Cohen JD (1999) Conflict monitoring versus selection-for-action in anterior cingulate cortex. Nature 402:179-181. CrossRef Medline

Caspers S, Eickhoff SB, Rick T, von Kapri A, Kuhlen T, Huang R, Shah NJ, Zilles K (2011) Probabilistic fibre tract analysis of cytoarchitectonically defined human inferiorparietal lobule areas reveals similarities to macaques. Neuroimage 58:362-380. CrossRef Medline

Corbetta M, Patel G, Shulman GL (2008) The reorienting system of the human brain: from environment to theory of mind. Neuron 58:306-324. CrossRef Medline

den Ouden HE, Daunizeau J, Roiser J, Friston KJ, Stephan KE (2010) Striatal prediction error modulates cortical coupling. J Neurosci 30:32103219. CrossRef Medline

Deubel H (2008) The time course of presaccadic attention shifts. Psychol Res 72:630-640. CrossRef Medline

Dombert PL, Kuhns A, Mengotti P, Fink GR, Vossel S (2016a) Functional mechanisms of probabilistic inference in feature- and space-based attentional systems. Neuroimage 142:553-564. CrossRef Medline

Dombert PL, Fink GR, Vossel S (2016b) The impact of probabilistic feature cueing depends on the level of cue abstraction. Exp Brain Res 234:685694. CrossRef Medline

Egner T, Monti JM, Trittschuh EH, Wieneke CA, Hirsch J, Mesulam MM (2008) Neural integration of top-down spatial and feature-based information in visual search. J Neurosci 28:6141-6151. CrossRef Medline

Eickhoff SB, Stephan KE, Mohlberg H, Grefkes C, Fink GR, Amunts K, Zilles K (2005) A new SPM toolbox for combining probabilistic cytoarchitectonic maps and functional imaging data. Neuroimage 25:1325-1335. CrossRef Medline

Friston KJ, Holmes AP, Worsley KJ, Poline JP, Frith CD, Frackowiak RS (1995) Statistical parametric maps in functional imaging: a general linear approach. Hum Brain Mapp 2:189-210.

Friston KJ, Williams S, Howard R, Frackowiak RS, Turner R (1996) Movement-related effects in fMRI time-series. Magn Reson Med 35:346355. CrossRef Medline

Friston KJ, Buechel C, Fink GR, Morris J, Rolls E, Dolan RJ (1997) Psycho- physiological and modulatory interactions in neuroimaging. Neuroimage 6:218-229. CrossRef Medline

Gitelman DR (2002) ILAB: A program for postexperimental eye movement analysis. Behav Res Methods Instrum Comput 34:605-612. CrossRef Medline

Harrison LM, Duggins A, Friston KJ (2006) Encoding uncertainty in the hippocampus. Neural Netw 19:535-546. CrossRef Medline

Jocham G, Neumann J, Klein TA, Danielmeier C, Ullsperger M (2009) Adaptive coding of action values in the human rostral cingulate zone. J Neurosci 29:7489-7496. CrossRef Medline

Lasaponara S, Chica AB, Lecce F, Lupianez J, Doricchi F (2011) ERP evidence for a selective drop in attentional costs in uncertain environments: challenging a purely premotor account of covert orienting of attention. Neuropsychologia 49:2648-2657. CrossRef Medline

Mathys C, Daunizeau J, Friston KJ, Stephan KE (2011) A Bayesian foundation for individual learning under uncertainty. Front Hum Neurosci 5:39. CrossRef Medline

McLaren DG, Ries ML, Xu G, Johnson SC (2012) A generalized form of context-dependent psychophysiological interactions (gPPI): a comparison to standard approaches. Neuroimage 61:1277-1286. CrossRef Medline

Oldfield RC (1971) The assessment and analysis of handedness: the Edinburgh inventory. Neuropsychologia 9:97-113. CrossRef Medline

Posner MI, Snyder CR, Davidson BJ (1980) Attention and the detection of signals. J Exp Psychol 109:160-174. CrossRef Medline

Ranganath C, Ritchey M (2012) Two cortical systems for memory-guided behaviour. Nat Rev Neurosci 13:713-726. CrossRef Medline

Rushworth MF, Nixon PD, Renowden S, Wade DT, Passingham RE (1997) The left parietal cortex and motor attention. Neuropsychologia 35:12611273. CrossRef Medline

Rushworth MF, Ellison A, Walsh V (2001a) Complementary localization and lateralization of orienting and motor attention. Nat Neurosci 4:656661. CrossRef Medline

Rushworth MF, Krams M, Passingham RE (2001b) The attentional role of the left parietal cortex: the distinct lateralization and localization of motor attention in the human brain. J Cogn Neurosci 13:698-710. CrossRef Medline

Seghier ML (2013) The angular gyrus: multiple functions and multiple subdivisions. Neuroscientist 19:43-61. CrossRef Medline

Stephan KE, Penny WD, Daunizeau J, Moran RJ, Friston KJ (2009) Bayesian model selection for group studies. Neuroimage 46:1004-1017. CrossRef Medline

Strange BA, Duggins A, Penny W, Dolan RJ, Friston KJ (2005) Information theory, novelty and hippocampal responses: unpredicted or unpredictable? Neural Netw 18:225-230. CrossRef Medline

Tobia MJ, Gläscher J, Sommer T (2016) Context-specific behavioral surprise is differentially correlated with activity in anterior and posterior brain systems. Neuroreport 27:677-682. CrossRef Medline

Tzourio-Mazoyer N, Landeau B, Papathanassiou D, Crivello F, Etard O, Delcroix N, Mazoyer B, Joliot M (2002) Automated anatomical labeling of activations in SPM using a macroscopic anatomical parcellation of the MNI MRI single-subject brain. Neuroimage 15:273-289. CrossRef Medline

van Gaal S, Ridderinkhof KR, Scholte HS, Lamme VA (2010) Unconscious activation of the prefrontal no-go network. J Neurosci 30:4143-4150. CrossRef Medline

Viard A, Doeller CF, Hartley T, Bird CM, Burgess N (2011) Anterior hippocampus and goal-directed spatial decision making. J Neurosci 31:46134621. CrossRef Medline

Vossel S, Mathys C, Daunizeau J, Bauer M, Driver J, Friston KJ, Stephan KE (2014a) Spatial attention, precision, and Bayesian inference: a study of saccadic response speed. Cereb Cortex 24:1436-1450. CrossRef Medline

Vossel S, Bauer M, Mathys C, Adams RA, Dolan RJ, Stephan KE, Friston KJ (2014b) Cholinergic stimulation enhances Bayesian belief updating in the deployment of spatial attention. J Neurosci 34:15735-15742. CrossRef Medline

Vossel S, Mathys C, Stephan KE, Friston KJ (2015) Cortical coupling reflects Bayesian belief updating in the deployment of spatial attention. J Neurosci 35:11532-11542. CrossRef Medline 